\title{
The effect of intravesical Marcain instillation on hyperreflexic detrusor contractions
}

\author{
P D McInerney MBChB FRCS, ${ }^{1}$ A Grant MB BCh, ${ }^{2} \mathrm{~J}$ Chawla MD FRCS, ${ }^{2} \mathrm{~T}$ P \\ Stephenson MS FRCS ${ }^{1}$ \\ ${ }^{1}$ Department of Urology, Cardiff Royal Infirmary, Newport Road, Cardiff CF2 1SZ, \\ South Wales; ${ }^{2}$ Rookwood Hospital, Cardiff, South Wales.
}

Thirty-six patients with suprasacral spinal injury were treated with intravesical local anaesthetic instillation. Eighteen of 32 patients $(56 \%)$ were converted from an 'ice water positive' hyperreflexic state to an 'ice water negative' state by bupivicaine hydrochloride. Four patients treated with lignocaine hydrochloride showed no benefit. Intravesical local anaesthetic instillation is suggested as a possible treatment for selected cases of detrusor hyperreflexia in patients on intermittent catheterisation.

Key words: local anaesthetic; detrusor hyperreflexia; spinal injury.

\section{Introduction}

After recovery from 'spinal shock' during which the detrusor remains acontractile, suprasacral spinal injured patients usually develop involuntary detrusor contractions (hyperreflexia), which are almost invariably related to some degree of detrusor-sphincter dyssynergia. ${ }^{1}$ Provocative tests during filling cystometry help to confirm such contractions. The 'ice water fill' is such a test ${ }^{2}$ and, although it has fallen into disfavour in recent years, our own experience has shown little difference between detrusor and urethral pressure measurement during rapid ice water filling cystometry and conventional slow fill cystometry. Iced water provides a sensory stimulus and the efficacy of the test suggests that there is an afferent arm to the hyperreflexic contraction.

Previous studies have given conflicting results on the influence of local anaesthetics on detrusor contractility. 3,4

There has been some preliminary work on manipulating the motor side of the reflex contraction with intravesical anticholinergic therapy ${ }^{5}$ but there is very little published data on the influence of the sensory side of the reflex.

Early suggestions that the reflex was initiated at sphincter or pelvic floor level have not been substantiated. ${ }^{6}$ Specific cold receptors have been identified in the blad- der. ${ }^{7,8}$ Stretch receptors are also postulated.

The ability of local anaesthetic instillation to influence hyperreflexic detrusor contractions has been studied.

\section{Patients and methods}

Thirty-six patients (aged 18-68) with symptoms suggestive of detrusor hyperreflexia after suprasacral spinal injury were studied. There were 31 males and 5 females, including 20 patients with cervical injuries and 16 with thoracic injuries. Assessment was conducted between 12 weeks and 57 years post injury. All underwent filling cystometry.

A $10 \mathrm{FG}$ filling catheter and a $5 \mathrm{FG}$ Gaeltec catheter carrying a single bladder pressure transducer at the tip and 2 urethral transducers were passed transurethrally. The optimum position with the distal transducer in the bladder and the proximal transducers at the point of maximum urethral pressure was monitored both by pressure recording and radiologically.

The bladder was emptied and the resting pressures recorded. Provocative filling at a rate of $100 \mathrm{ml}$ per minute using $100 \mathrm{ml}$ of ice cold saline and urografin contrast medium was commenced and the detrusor and urethral pressure responses recorded. The test was regarded as positive if spontaneous voiding occurred around the filling catheter 
in response to a rise in bladder pressure. The outflow tract was studied radiologically during involuntary voiding (Fig 1). The bladder was emptied and local anaesthetic instillation was performed. In 4 patients $40 \mathrm{ml} 1 \%$ Lignocaine was used but the remainder all had $40 \mathrm{ml} 0.5 \%$ Marcain (bupivicaine hydrochloride) instilled and left intravesically for 15 minutes, before the bladder was re-emptied. The ice water test was then repeated. Because of potential autonomic dysreflexia, blood pressure was monitored throughout the study.

\section{Results}

Only patients who were ice water positive were included. Lignocaine instillation in 4 patients failed to prevent the hyperreflexic response to the ice water contraction and in each case a more brisk and powerful contraction was seen after the Lignocaine, suggesting that the bladder might be sensitised by the dosage used.

In the remaining 32 patients, Marcain instillation was employed and in 18 cases $(56 \%)$ resulted in suppression of the re-

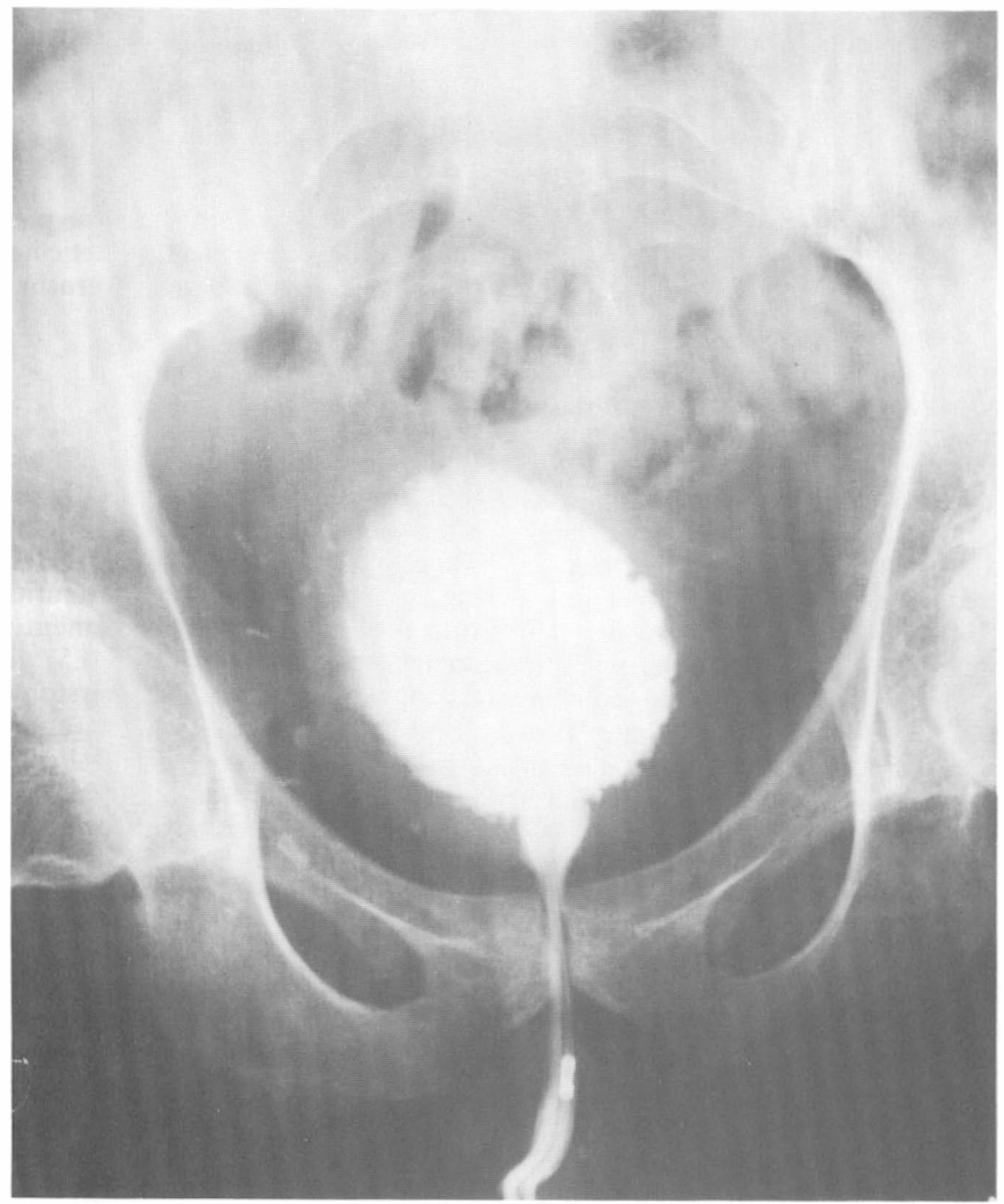

Figure 1 Radiograph showing neuropathic bladder during involuntary voiding. Pressure transducer lies at the lower margin of sphincter activity. 
sponse to ice water (Fig 2). Eight of the remaining 14 patients showed a delay in the onset of the provoked contraction. No ureteric reflux was demonstrated radiologically.

No pattern was established to help predict which patients might respond best to local anaesthetic instillation. The responders included 8 midthoracic and 10 cervical lesions and there was coincidentally an identical distribution in the passive group. In patients where Marcain instillation was successful, detrusor pressures generated in response to the ice water test were suppressed by between 20 and $50 \mathrm{~cm} \mathrm{H}_{2} \mathrm{O}$. In 7 of these patients there was a maximum detrusor pressure of less than $4 \mathrm{~cm} \mathrm{H}_{2} \mathrm{O}$ during ice water filling.

All patients exhibited a degree of detrusor sphincter dyssynergia on cystometry. Local anaesthetic instillation did not appear to influence the urethral pressure response.

The duration of the response to Marcain was not routinely studied, but 2 patients who had responded were tested three hours

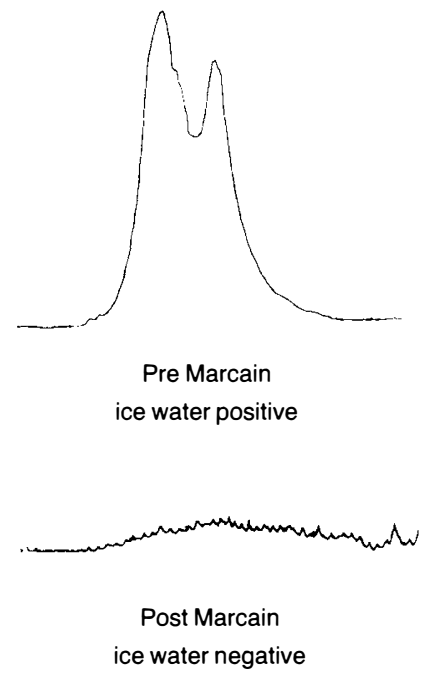

Figure 2 Urodynamic tracing showing an ice water positive response converted to an ice water negative response by Marcain instillation. after instillation and had remained ice water negative.

\section{Discussion}

Previous studies have examined the effect of Lignocaine on detrusor instability and bladder capacity and have either shown lowering of maximum detrusor pressure ${ }^{4}$ or worsening of instability in patients with outflow obstruction. ${ }^{9}$ With only 4 patients in the study it is difficult to assess accurately the effect of Lignocaine but the findings concurred with those of the latter study. It may be that the dose of Lignocaine employed was too low. Previous studies using $2 \%$ Lignocaine have shown a better response. ${ }^{8}$ Marcain on the other hand does not appear to sensitise the bladder to provocative stimuli.

Intravesical local anaesthesia with Marcain has been used in the treatment of autonomic dysreflexia but not of detrusor hyperreflexia.

The permeability of bladder mucosa is well known and although this study shows that local anaesthetic instillation may dampen down detrusor contractions, it is not evident from the study if the result is due to blockade of the afferent arm of the cooling reflex, or to detrusor paralysis from Marcain permeating into the muscle layer through the mucosa. The cases where the ice water contraction was delayed, but remained, tend to suggest that sensory blockade is the more likely mechanism.

The reported success of intravesical anticholinergic therapy ${ }^{5}$ offers relief for patients on intermittent catheterisation who are wet between catheters. The systemic side effects however of anticholinergics are well known, and the use of local anaesthesia in a similar fashion appears to provide an effective alternative although its duration of efficacy, serum level and side effect profile should be more extensively investigated before more widespread use is advocated.

\section{References}

1 Bary PR, Day G, Lewis P, Chawla J, Evans C, Stephenson TP (1982) Dynamic urethral function in the assessment of spinal injury patients. Br J Urol 54: 39-44.

2 Bors E (1957) Neurogenic bladder. Urol Surv 7: 177-250. 
3 Bors E, Binn KA (1957) Spinal reflex activity from the vesical mucosa in paraplegic patients. Am Arch Neurol Psychiatry 78: 339-354.

4 Higson RH, Smith JC, Hills W (1979) Intravesical Lignocaine and detrusor instability. Br J Urol 51: 500-503.

5 Madersbacher H (1990) The various types of neurogenic bladder dysfunction: an update of current therapeutic concepts. Paraplegia 28: 217-229.

6 Sutherst JR, Brown M (1978) The effect on the bladder pressure of sudden entry of fluid into the posterior urethra. Br J Urol 50: 406-409.

7 Fall M, Lindstrom S, Maziers L (1987) Experimental aspects of the bladder cooling reflex. Neuro-urol Urodyn 6: 228.

8 Lindstrom S, Maziers L, Fall M (1988) The bladder cooling reflex: characteristics of afferent mechanism. Neuro-urol Urodyn 7: 248.

9 Sethia KK, Smith JC (1987) The effect of $\mathrm{pH}$ and Lignocaine on detrusor instability. Br J Urol 60: 516-518. 